\title{
BMJ Open Generating national projections of dementia cases for Ireland using a calibrated macro-simulation model
}

\author{
Tom Pierse (1) , ${ }^{1}$ Fiona Keogh, ${ }^{1}$ Stephen O'Neill ${ }^{2,3}$
}

To cite: Pierse T, Keogh F, O'Neill S. Generating national projections of dementia cases for Ireland using a calibrated macrosimulation model. BMJ Open 2020;10:e035463. doi:10.1136/ bmjopen-2019-035463

- Prepublication history and additional material for this paper are available online. To view these files, please visit the journal online (http://dx.doi. org/10.1136/bmjopen-2019035463).

Received 01 November 2019 Revised 04 June 2020 Accepted 26 June 2020

Check for updates

(C) Author(s) (or their employer(s)) 2020. Re-use permitted under CC BY-NC. No commercial re-use. See rights and permissions. Published by BMJ.

${ }^{1}$ Centre for Economic and Social Research on Dementia, National University of Ireland, Galway, Ireland

${ }^{2}$ Department of Health Services Research and Policy, London School of Hygiene \& Tropical Medicine, London, United Kingdom

${ }^{3}$ J.E. Cairnes School of Business and Economics, National University of Ireland, Galway, Ireland

Correspondence to

Dr Tom Pierse;

tom.pierse@nuigalway.ie

\section{ABSTRACT}

Introduction Epidemiological data on dementia is not available in many European countries and regions due to the high cost and complexity of conducting large scale dementia screening studies. The available epidemiological studies identify potentially substantial variation in the prevalence of dementia over time and across Europe. Methods In this paper we generate simulations of the number of dementia cases in Ireland from 1991 to 2036 using a three-state Markov illness-death model. Parameters values are selected for each simulation from a range using a random parameter search pattern. We employ a novel calibration method which exploits the strong relationship between dementia, ageing and mortality. Simulation weights are generated based on differences between observed and modelled cohorts of older people and the reported number of deaths from dementia. Irish Census data from 1991 to 2016 and the number of recorded deaths due to dementia in 2018 are used as calibration points. A weighted average projection of the number of dementia cases is generated.

Results We estimate a weighted average number of cases of dementia in 2016 of 54877 increasing to 98 946 in 2036; this estimate is substantially lower than the estimates generated using extrapolation methods. We show the wide range of possible outcomes given the range in the available parameter estimates and show that irrespective of whether the incidence rate of dementia is declining the number of cases of dementia is rapidly increasing due to population ageing

Conclusion Previous studies have used parameter estimates from meta-analyses of the literature or from individual studies. In this paper we supplement these with a calibration approach using observed cause of death and population age structure data. These additional sources of data can be used to generate estimates of dementia prevalence in any country or region which has census data and data on deaths due to dementia.

\section{INTRODUCTION}

Dementia is a chronic or progressive syndrome characterised by deterioration in memory, thinking, behaviour and the ability to perform everyday activities. ${ }^{1}$ The number of people living with dementia continues to increase, currently the condition affects an estimated 50 million people worldwide with a global cost estimated to reach US $\$ 1$ trillion

\section{Strengths and limitations of this study}

This study demonstrates a method for generating robust estimates of the number of cases of dementia that can be applied to any country or region with census data and cause of death data.

- The study provides estimates and projections of the number of cases of dementia in Ireland that are consistent with available Irish data.

- We show the growth and the credible range in the number of cases of dementia for the projection period. These are key requirements for planning future healthcare needs.

- The quality of data on dementia as a cause of death in Ireland is currently poor; this study provides a motivation for gathering data on dementia as a cause of death.

- While we have used the best available epidemiological data from the literature, the results may be sensitive to the modelling assumptions.

in $2018 .^{2}$ People with dementia, particularly moderate-to-severe dementia, have extensive health and social care needs. ${ }^{3}$

For policymakers to plan for current and future needs, guidance on the number of people with dementia and their care needs is required. However, many countries have little or no epidemiological data on dementia. ${ }^{4}$ Due to the substantial cost of large scale screening studies, many countries are likely to remain without local, population representative prevalence estimates in the foreseeable future. ${ }^{5}$ Pragmatic solutions are required which would exploit available national data to generate robust estimates of dementia prevalence for service planning purposes, particularly given the increasing prevalence of dementia and the challenge posed to the sustainability of health systems by chronic conditions. ${ }^{6}$

Many of the European epidemiological studies of dementia were carried out in the $1980 \mathrm{~s}$ and 1990s. ${ }^{7}$ There are indications from a small number of more recent large scale studies that incidence (the number of 
new cases per year) and prevalence rates for dementia (the total number of cases) may have declined. ${ }^{8}$ Though comparison is difficult due to differences in study methods, there is some evidence that prevalence rates may vary between countries and regions of the world. ${ }^{9}$ Variation in prevalence rates have also been shown within large countries, ${ }^{1011}$ a recent study using German administrative data demonstrated the wide variation in the prevalence of dementia within Germany-with age standardised prevalence rates of between 6 and 10 per cent of people over 65 years of age. ${ }^{11}$

A range of risk factors for dementia have been identified including: hearing loss, education level, genetics (APOE e4 gene), smoking, depression, social isolation, physical activity, diabetes, obesity and hypertension; in addition to age. ${ }^{12}$ The prevalence of these risk factors varies widely across Europe. For example, the prevalence of the APOE e4 gene, is higher in the Scandinavian countries and lower in southern Europe ${ }^{13}$ Other than age, mid-life hearing loss is potentially the most important risk factor for dementia. ${ }^{12}$ While wide variation in rates of hearing loss have been found across countries, methodological consistency in this area is low. ${ }^{14}$ However, how the risk factors of dementia are inter-related and the mechanisms through which they alter the incidence and prevalence of dementia are not well understood. Improvements in education and reductions in smoking and hypertension may be influencing the reduction in the incidence of dementia despite increases in obesity and physical inactivity in many countries. ${ }^{1516}$ What is clear is that there is a significant potential for cross-country and within-country regional differences in prevalence rates of dementia.

The prevalence of dementia in Ireland currently used for service planning has been calculated using a metaanalysis of international studies resulting in an estimate of 55000 people with dementia. ${ }^{17}$ These estimates have been used to establish rates of provision for different services in Ireland and to examine regional differences to guide future resource allocation. ${ }^{18}$ However, if prevalence rates from a recent large scale study in the UK ${ }^{19}$ were to be used, these estimates would be substantially lower. ${ }^{20}$ The national dementia strategy calls for improved national estimates of current and future prevalence of dementia. ${ }^{21}$ More sophisticated estimates would facilitate scenario planning for future capacity. ${ }^{22}$ Such scenarios could explore the potential for responding to public preferences for home care ${ }^{2324}$ and examine, for example, the optimal balance of home care and residential care while responding to increases in the number of people with dementia.

In this paper we generate a set of simulations based on international epidemiological data from 1991 to 2036. By generating simulations based on parameter ranges rather than point estimates we demonstrate the potential range of the number of cases of dementia in Ireland based on the available evidence, which facilitates more accurate planning, and scenario-based planning for future resource allocation. A novel contribution of this paper is to calibrate these simulations using Irish census data and the number of deaths due to dementia in 2018. This allows us to estimate a weighted average number dementia cases for the projection period that are consistent with the available Irish data.

\section{METHODS}

Projections of the number of people with dementia have previously been carried out in Ireland and elsewhere by multiplying historical population dementia prevalence rates by future population projections for each agegender group..$^{25}$ This extrapolation method benefits from being simple, however it may lead to biassed projections if the age and gender specific prevalence rates vary over time. ${ }^{26}$ For example, if incidence rates are in decline this would not be captured in historical prevalence studies. In addition, it is not clear what prevalence rates should be used; in the Irish context this could, for instance, be data from an international meta-analysis ${ }^{27}$ or the most recent UK study. ${ }^{19}$ While many of the behavioural risk factors in Ireland are likely to be similar to those of the UK, we cannot be confident that the major risk factors are well aligned, leading to biassed projections. We know relatively little about hearing loss in different areas, there are educational differences between Ireland and the UK and the prevalence of the APOE e4 gene in Ireland is not available.

An alternative approach to generating projections is by macro-simulation. This involves modelling the aggregate flows of older people between age groups and dementia states. ${ }^{28}$ A key advantage of macro-simulation over the simpler extrapolation approach is that more nuanced alternative scenarios of changing incidence and mortality can be considered.

Macro-simulations, to generate the projected number of dementia cases, have been carried out for the USA ${ }^{29}$ and a number of European countries. ${ }^{30}{ }^{31}$ However, none of these papers have used census data to calibrate their projections. Generating a macro-simulation model of dementia prevalence rates requires assumptions about the initial prevalence of dementia in the population; incidence rates over the study period; and mortality rates for both the dementia and non-dementia populations. For this exercise, these are sourced from the international literature (outlined below) as there have been no large scale epidemiological studies of dementia in Ireland.

\section{Model overview}

A multi-state Markov illness-death model is used to simulate the number of people in all age cohorts in each of three states-no dementia, dementia and dead. ${ }^{32} 33$ The flows between these states are determined by the number of people in a state and the transition probabilities between states. Transition probabilities for dementia incidence and mortality (from dementia and non-dementia causes) vary by year, age and gender. The total number of people in age cohort $i$ at time $t$, denoted by $\mathrm{C}_{\mathrm{it}}$, is 
calculated as: $C_{i t}=N_{i t}+N_{i t}^{*}$, where $N\left(N^{*}\right)$ is the number of people in that cohort without (with) dementia. The number of people in the non-dementia age cohort $i$ in period $t$ is determined by the number of individuals in that cohort in the preceding period, adjusted by the probability of individuals having died in that period $\left(M_{i t-1}\right)$, and the incidence rate for dementia $\left(I_{i t-1}\right)$, defined as the number of new dementia cases in the age cohort over the number of individuals in the cohort in that year.

$$
N_{i t}=N_{i t-1}\left(1-M_{i t-1}-I_{i t-1}\right)
$$

Non-dementia mortality is modelled as an exponential function with a separate time trend for the historical and the projection periods.

$$
M_{i t-1}=\exp \left(M_{\text {Intercept }}+M_{\text {Age }} * i+M_{t r 1} * t_{1}+M_{t r 2} * t_{2}\right)
$$

Where $M_{\text {Intercept }}$ and $M_{\text {Age }}$ are coefficients of the base mortality model. $M_{t r 1}$ is the trend for the 1991 to 2016 period $\left(\mathrm{t}_{1}\right)$ and $M_{t r 2}$ is the trend in the projection period $\left(\mathrm{t}_{2}\right)$. The number of people in the dementia age cohort $i$ in period $t$ is determined by the number of individuals in that cohort in the preceding period reduced by individuals having died in that period, and increased by the number of new dementia cases. The probability of mortality in the dementia state is the product of the non-dementia mortality rate $\left(M_{i t-1}\right)$ and the mortality rate of people with dementia relative to the mortality rate of people without dementia $\left(\mathrm{RM}_{\mathrm{i}}\right) \cdot \mathrm{RM}_{\mathrm{i}}$ decays with age at a rate of $\mathrm{RM}_{\text {Decay }} \mathrm{RM}_{65}$ and $\mathrm{RM}_{\text {Decay }}$ are linked such that a high relative mortality rate at 65 years of age coincides with a higher rate of decay.

$$
\begin{gathered}
N_{i t}^{*}=N_{i t-1}^{*}\left(1-R M_{i} * M_{i t-1}\right)+N_{i t-1} * I_{i t-1} \\
R M_{i}=R M_{65} *\left(1-R M_{\text {Decay }}\right)^{i}
\end{gathered}
$$

The incidence of dementia has been shown to exhibit exponential growth with increasing age. ${ }^{34}$ Incidence is modelled as follows:

$$
I_{i t-1}=I_{65} *\left(1+I_{t r}\right)^{t} *\left(1+I_{\text {growth }}\right)^{i}
$$

Where $I_{65}$ is the incidence of dementia at 65 years of age in 1991, $I_{t r}$ is the trend in $I_{65}$ over time and $I_{g r o w t h}$ is the increase in incidence rates with age. Together, equations 1 to 5 allow the model to project the number of individuals in the dementia and non-dementia states over time, given a number of inputs which are discussed below. Five thousand simulations are generated for both men and women. Simulations are generated for single year-age cohorts of men and women between 65 and 98 years of age. The minimum and maximum ages of 65 years and 98 years are used as there is sparse available data on the incidence/prevalence of dementia for younger people with dementia and in the oldest old..$^{3536}$

Simulations were carried out on R (V.3.6) using the heemod package. ${ }^{33}$

\section{Model parameters}

Parameters are selected for each simulation from a distribution using a random parameter search pattern. ${ }^{37}$ This approach generates a set of parameters for each simulation by selecting values at random from a range provided for each parameter. The choice of potential parameter values for these inputs was informed by a review of the literature outlined below. The parameter ranges used are summarised in online supplementary table 1 . With the exception of the incidence rate, all parameter values are selected from a uniform distribution.

\section{Incidence rate at 65 in 1991}

International studies of incidence have produced a wide range of estimates. ${ }^{38}$ The incidence rate for men and women at aged 65 in 1991 is selected from a truncated normal distribution with a mean of 4.2 cases per 1000 , based on an interpolation of the 60 to 64 and 65 to 69 categories, ${ }^{2}$ a maximum of 8.2 cases per $1000^{39}$ and a minimum of 2.3 cases per $1000 .{ }^{40}$ A floor for incidence rate at $65\left(I_{65}\right)$ is set at 1.5 cases per 1000 to maintain a positive prevalence of dementia.

Increase in incidence with age $\left(I_{\text {growth }}\right)$

Incidence rates have been shown to double every 5.8 years, which is equivalent to an increase of 12 per cent in the incidence rate for every year of age. ${ }^{2}$ The search range for this parameter is from 11 to 13 per cent per year.

\section{Trend in incidence rates $\left(I_{t r}\right)$}

There are indications from a number of large scale studies that incidence rates have declined over time. In the US Framingham Heart Study, a decline in dementia incidence rates of 3 per cent per year was found. In the UK, the Cognitive Functioning and Ageing Study showed a decline in incidence rates between the early 1990s and the late 2000s of 20 per cent, or 2 per cent per year. ${ }^{39}$ A decline in incidence may be due to reduced exposure to dementia risk factors (eg, healthier lifestyles) and/or increased exposure to protective factors (such as education) (ibid). The possible effect of preventative measures is operationalised in the paper through a decay in the incidence rate of between 0 and 2 per cent per calendar year.

Historical non-dementia mortality $\left(M_{\text {Intercept }} M_{\text {Age }} M_{t r 1}\right)$

Aggregate mortality is used to identify the search range for non-dementia mortality. A set of 1000 simulations are run initially with the dementia prevalence and incidence set to zero. The parameters for the best fitting simulations for both men and women were chosen using the calibration process described below, and were used as the basis for generating the non-dementia mortality rates.

\section{Projected non-dementia mortality $\left(M_{t r 2}\right)$}

The main assumption underlying the projections for the number of older people in Ireland is the rate of decline in mortality. While fertility and migration may have a significant impact on population projections for younger age cohorts, the population of older people is not as sensitive to assumptions about fertility and migration over a 
20-year horizon. ${ }^{41}$ The trend in mortality rates used here range from a continuation of the historical trend to trend declining to no yearly improvement over the projection period.

\section{Dementia relative mortality $\left(R M_{i}\right)$}

The mortality rates of people with dementia have been estimated in incidence studies to be in the region of twice those of non-dementia patients. ${ }^{42-45}$ A number of studies have shown variation in the relative mortality for different age groups. Relative mortality rates are elevated for younger dementia cases with little difference in the oldest age categories. ${ }^{28} 424647$ In this study, the relative mortality for 65 year old age cohorts is selected from a range of 2 to $5^{28}$, and allowed to decay with age such that the overall relative mortality rate for people with dementia is twice that of the non-dementia population. We assume that the relationship between dementia and non-dementia mortality $\mathrm{RM}_{\mathrm{i}}$ is allowed to vary by age but is constant over time.

\section{Data}

All historical population data, including data on the number and gender of people turning 65 each year are sourced from the Irish census for 1991, 1996, 2001, 2006, 2011 and 2016. Population estimates for new cohorts of 65 year olds are sourced from the Central Statistics Office projections, as are the number of deaths registered as being caused by dementia in $2018 .^{41}$

\section{Calibration}

With the procedure described above we generate 5000 simulations for men and women, both with and without dementia. Two approaches are used to attach greater weight to simulations that are more likely to reflect reality. First, all simulations where the number of deaths due to dementia are less than the number recorded in 2018 are excluded. While the recorded number of deaths attributable to dementia in Ireland is likely to be substantially below the true figure, due to low rates of diagnosis, using this data allows us to set a floor on dementia estimates.

Second, we weight the remaining simulations based on the difference between the simulated and observed census populations. A squared error term is generated by summing the squared error on the age distribution for each of the age categories from 65 to 98 in each of the five census years (1996, 2001, 2006, 2011 and 2016).

$$
\text { error }^{2}=\sum_{t=1}^{t=5} \sum_{i=65}^{i=98}\left(C_{i t}-\hat{C}\right)_{i t}^{2}
$$

Where $C_{i t}$ and $C_{i t}$ are the number of people, in a 1 year age category, in the census and in the simulation, respectively. A weight for each of the $J$ simulations $(j=1, \ldots J)$ is generated as follows.

$$
\text { weight }_{j}=\frac{1}{\text { error }_{j}^{2}} * \sum_{j=1}^{J} \text { error }_{j}^{2}
$$

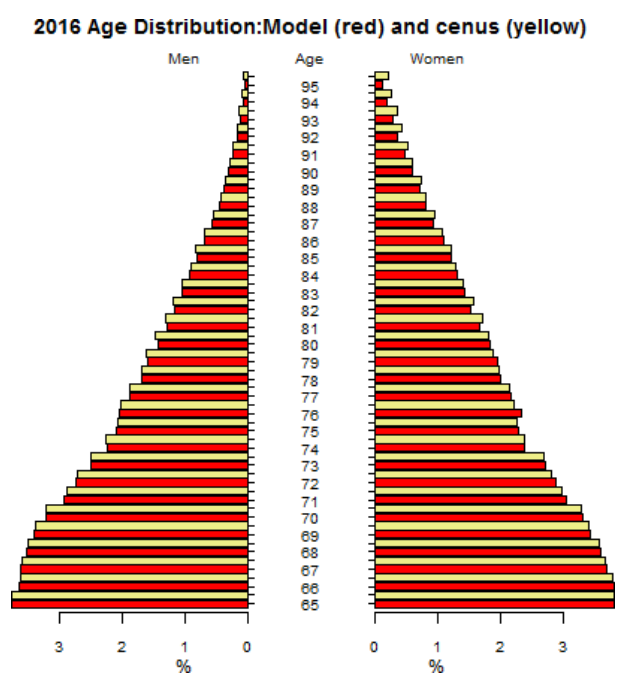

Figure 1 Population age distribution for 2016 for the simulation with the lowest error. Simulation estimates are shown in red and the observed census data are shown in yellow.

A weighted average number of dementia cases can then be generated using these weights. To show the possible range of trajectories, a fan plot is generated based on the simulations with the lowest error.

\section{Patient and public involvement}

People with dementia, through participation on the steering committee and the dementia advisory panel of the Centre for Economic and Social Research on Dementia, are involved in directing the programme of research of which this study is a component.

\section{RESULTS}

\section{Model fit}

The calibration approach used here allows us to identify the observed sets of parameters $\left(M_{\text {Intercept }}, M_{\text {Coof }}, M_{t r 1}, M_{t r 2}, R M_{i}, I_{65}, I_{\text {growth }}, I_{t r}\right)$ that are most consistent with the observed Irish demographic data. Figure 1 show the population age distribution for 2016 for the model with the lowest error from the full set of simulations. This shows that the best fitting simulations are closely aligned with the census for 2016 .

\section{Projected number of cases of dementia}

Figure 2 shows a fan plot of the number of people with dementia based on 100 simulations with the lowest calibration error for men and women (combined). The weighted average estimate of the number of cases of dementia for 2016 is 54877 expanding to 98946 by 2036, as shown in table 1 . This latter projection for 2036 is substantially lower than previous projections (124 735) based on an extrapolation methodology. ${ }^{25}$ However, the plot shows the wide range of possible outcomes given the range in the available parameter estimates. The plot also shows that irrespective of whether the incidence rate is declining the number of cases of dementia is rapidly 


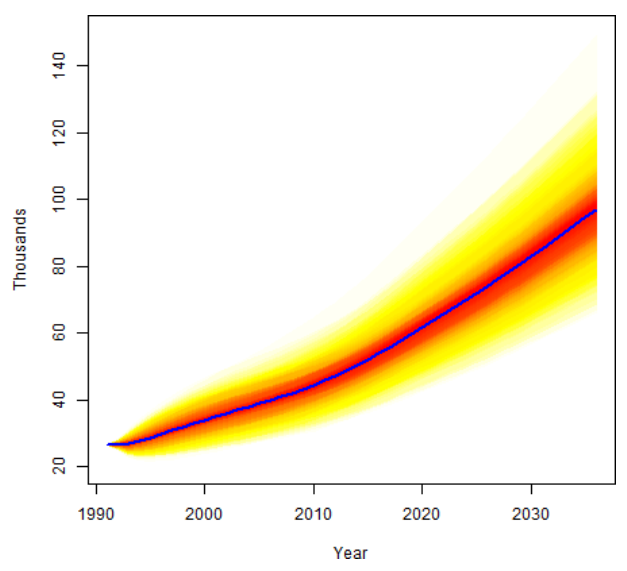

Figure 2 Fan plot of the estimated number of people with dementia. The weighted average of all simulations is shown in Blue. Colours depict percentiles of the distribution of the number of cases of dementia from the 100 simulations with the lowest calibration error.

increasing due to population ageing. A sensitivity analysis is provided in the online supplementary table 2.

\section{DISCUSSION}

The model developed in this paper demonstrates how census and cause of death data can be used to calibrate dementia projection models. We estimate a weighted average number of cases of dementia for Ireland of 54 887 in 2016, increasing to 98946 in 2036. The estimate for 2036 is substantially lower than that obtained in previous studies using extrapolation methodology.

A key difference between this study and the previous modelling studies of dementia cases is the use of census and cause of death data to calibrate models. ${ }^{293048}$ Previous studies have used parameter estimates from meta-analysis of the literature or from individual studies. In this paper we supplement these with a calibration approach. This approach allows additional sources of data such as census data and cause of death data to be used to customise the available estimates of dementia epidemiology in a country or region leading to more sensitive estimates. A second key difference between this study and previous modelling studies of dementia cases is the use of parameter ranges. Previous studies select a point estimate or show a limited number of scenarios. Using ranges rather than point estimates allows for the substantial uncertainty in many of the parameter estimates to be represented. ${ }^{49}$

The projections generated in this study differ from those currently available based on extrapolation in a number of ways. First, they are consistent with Irish demographic and cause of death data. Second, they incorporate plausible parameter ranges-for example the possible decline in the incidence rate over time. Third, they show the range in the probable number of cases of dementia.

There are a number of limitations to the data and methods in this study. Cause of death records in Ireland are likely to significantly under-report dementia as the primary cause as (i) the practice of recording dementia as a primary cause of death has only commenced in 2018 and (ii) due to low rates of diagnosis in Ireland. ${ }^{50}$ Improvements in the recording of dementia as a cause of death in Ireland would assist in eliminating scenarios with low estimated dementia incidence, prevalence and mortality thus improving the calibration of the model and narrowing the credible range of estimates. While the calibration procedure outlined in this paper can improve on an un-calibrated projection, care is still required in choosing the mean and distribution of the chosen parameters. The weighted average number of cases reported in table 1 will be influenced by the range and distribution of parameter values. A time trend in the relative mortality of people with dementia was not included in this study due to a lack of literature in this area to guide parameter selection. ${ }^{51}$ Dementia relative mortality could be increasing, for example, if people with dementia are not getting the benefits of technological improvements, or declining, if people with dementia are now receiving treatments that they would not have in the past due to changes in how people with dementia are perceived.

In the context of increasing prevalence of dementia globally and the targets set out in the WHO Global Action Plan on Dementia, ${ }^{52}$ countries are developing plans which will require investment in new service development and a greater quantum of existing services. The best possible incidence and prevalence estimates are required to aid this future planning. There are significant policy and care implications to using prevalence estimates that are too low or too high. For example, using an estimate for the number of people with dementia that was too low might suggest to service planners that there would not be sufficient demand for a service at a local level, for example, day care. ${ }^{53}$ Alternatively, using an estimate that was too high would overstate the cost of providing a new service, for example, providing post-diagnostic support to all newly diagnosed people with dementia. ${ }^{54}$

The methodology used in this study would be most effective in countries or regions with relatively frequent census, high rates of dementia diagnosis and high quality

Table 1 The weighted mean number of people with dementia from all simulations, aged 65 to 98 , for 2016 and projected census years

\begin{tabular}{|c|c|c|c|c|c|}
\hline & 2016 & 2021 & 2026 & 2031 & 2036 \\
\hline Weighted SD & (9804) & (11 769) & (13 763) & (16 020) & (18 320) \\
\hline
\end{tabular}


cause of death data. The next census in Ireland is due in 2021, this can be added to the calibration exercise to improve these estimates. The methodology used in this study could also be applied to other diseases, such as diabetes, or for other purposes such as budget impact analysis.

Contributors TP contributed to all stages of the study from conception to drafting; SON contributed to the study methods and drafting of the manuscript; FK contributed to the concept discussions and drafting of the manuscript.

Funding This study was funded under a Health Research Board Applied Partnership Award, grant no. APA-2016-1876.

\section{Competing interests None declared.}

Patient and public involvement Patients and/or the public were involved in the design, or conduct, or reporting, or dissemination plans of this research. Refer to the Methods section for further details.

Patient consent for publication Not required.

Provenance and peer review Not commissioned; externally peer reviewed.

Data availability statement Data are available in a public, open access repository. Historical and projected population data (M2F2 projection), and cause of death data, are available from the central statistics office at: https://statbank.cso.ie/. Simulation code is available from the authors on request.

Open access This is an open access article distributed in accordance with the Creative Commons Attribution Non Commercial (CC BY-NC 4.0) license, which permits others to distribute, remix, adapt, build upon this work non-commercially, and license their derivative works on different terms, provided the original work is properly cited, appropriate credit is given, any changes made indicated, and the use is non-commercial. See: http://creativecommons.org/licenses/by-nc/4.0/.

ORCID iD

Tom Pierse http://orcid.org/0000-0002-2154-271X

\section{REFERENCES}

1 WHO. Towards a dementia plan a WHO guide, 2018.

2 ADI. World Alzheimer Report 2015: The Global Impact of Dementia. London: Alzheimer's Disease International, 2015.

3 Åkerborg Örjan, Lang A, Wimo A, et al. Cost of dementia and its correlation with dependence. J Aging Health 2016;28:1448-64.

4 Misiak B, Cialkowska-Kuzminska M, Frydecka D, et al. European studies on the prevalence of dementia in the elderly: time for a step towards a methodological consensus. Int $J$ Geriatr Psychiatry 2013;28:1211-21.

5 Gordon DS, Carter H, Scott S. Profiling the care needs of the population with dementia: a survey in central Scotland. Int $J$ Geriatr Psychiatry 1997:12:753-9.

6 Orueta JF, Nuño-Solinis R, Mateos M, et al. Monitoring the prevalence of chronic conditions: which data should we use? BMC Health Serv Res 2012;12:365.

7 Bacigalupo I, Mayer F, Lacorte E, et al. A systematic review and meta-analysis on the prevalence of dementia in Europe: estimates from the Highest-Quality studies adopting the DSM IV diagnostic criteria. J Alzheimers Dis 2018;66:1471-81.

$8 \mathrm{Wu}$ Y-T, Fratiglioni L, Matthews FE, et al. Dementia in Western Europe: epidemiological evidence and implications for policy making Lancet Neurol 2016;15:116-24.

9 Prince M, Bryce R, Albanese E, et al. The global prevalence of dementia: a systematic review and metaanalysis. Alzheimers Dement 2013;9:63-75.

10 Wu Y-T, Ali G-C, Guerchet M, et al. Prevalence of dementia in mainland China, Hong Kong and Taiwan: an updated systematic review and meta-analysis. Int J Epidemiol 2018;47:709-19.

11 Teipel S, Fritze T, Ovari A, et al. Regional pattern of dementia and prevalence of hearing impairment in Germany. J Am Geriatr Soc 2015;63:1527-33.

12 Livingston G, Sommerlad A, Orgeta V, et al. Dementia prevention, intervention, and care. The Lancet 2017;390:2673-734.

13 Corbo RM, Scacchi R. Apoe allele distribution in the world Is APOE* 4 a 'thrifty'allele? Annals of human genetics 1999;63:301-10.

14 Roth TN, Hanebuth D, Probst R. Prevalence of age-related hearing loss in Europe: a review. Eur Arch Otorhinolaryngol 2011;268:1101-7.
15 Eurobarometer412. Sport and physical activity. Brussels European Commission; 2014

16 NCD Risk Factor Collaboration (NCD-RisC). Trends in adult bodymass index in 200 countries from 1975 to 2014: a pooled analysis of 1698 population-based measurement studies with $19 \cdot 2$ million participants. Lancet 2016;387:1377-96.

17 O'Shea E, Cahill S, Pierce M. Developing and Implimenting Dementia Policy in Ireland. Galway: NUI Galway, 2017.

18 Keogh F, Pierse T, O'Shea E. Dementia services in Ireland 2018: Audit of community-based health and social care services used by people with dementia (forthcoming. Galway: NUI Galway, 2020.

19 Matthews FE, Bennett $\mathrm{H}$, Wittenberg R, et al. Who lives where and does it matter? changes in the health profiles of older people living in long term care and the community over two decades in a high income country. PLoS One 2016;11:e0161705.

20 Pierse T, Shea EO, Carney P. Estimates of the prevalence, incidence and severity of dementia in Ireland.. Ir J Psychol Med 2018.

21 Department of Health. The Irish national dementia strategy. Dublin: Department of Health, 2014.

22 Department of Health. Health service capacity review. Dublin: Department of Health, 2018.

23 Brown M. Responding to the Support \& Care Needs of our Older Population. Dublin: Sage, 2016.

24 Walsh S, O'Shea E, Pierse T, et al. Public preferences for home care services for people with dementia: a discrete choice experiment on personhood. Soc Sci Med 2020;245:112675.

25 Pierce M, Cahill S, O'Shea E. Planning dementia services: new estimates of current and future prevalence rates of dementia for Ireland. Ir J Psychol Med 2013;30:13-20.

26 Norton S, Matthews FE, Brayne C. A commentary on studies presenting projections of the future prevalence of dementia. BMC Public Health 2013;13:1.

27 Alzheimer Europe. Eurocode: report of WP 72006 prevalence of dementia in Europe, 2009.

28 Manuel DG, Garner R, Finès P, et al. Alzheimer's and other dementias in Canada, 2011 to 2031: a microsimulation population health modeling (POHEM) study of projected prevalence, health burden, health services, and caregiving use. Popul Health Metr 2016;14:37.

29 Brookmeyer R, Gray S, Kawas C. Projections of Alzheimer's disease in the United States and the public health impact of delaying disease onset. Am J Public Health 1998;88:1337-42.

30 Mura T, Dartigues J-F, Berr C. How many dementia cases in France and Europe? alternative projections and scenarios 2010-2050. Eur J Neurol 2010;17:252-9.

31 Lewis F. Estimation of future cases of dementia from those born in 2015. consulting report. London: Office of Health Economics, 2015.

32 Commenges D, Joly P, Letenneur L, et al. Incidence and mortality of Alzheimer's disease or dementia using an illness-death model. Stat Med 2004;23:199-210.

33 Filipović-Pierucci A, Zarca K, Durand-Zaleski I. Markov Models for Health Economic Evaluations: The R Package heemod, 2017.

34 Jorm AF, Jolley D. The incidence of dementia: a meta-analysis. Neurology 1998:51:728-33.

35 Lucca U, Tettamanti M, Logroscino G, et al. Prevalence of dementia in the oldest old: the Monzino 80-plus population based study. Alzheimers Dement 2015;11:258-70.

36 Lambert MA, Bickel H, Prince M, et al. Estimating the burden of early onset dementia; systematic review of disease prevalence. Eur $J$ Neurol 2014:21:563-9.

37 Bergstra J, Bengio Y. Random search for hyper-parameter optimization. J Mach Learn Res 2012;13:281-305.

38 Ziegler-Graham K, Brookmeyer R, Johnson E, et al. Worldwide variation in the doubling time of Alzheimer's disease incidence rates. Alzheimers Dement 2008;4:316-23.

39 Matthews FE, Stephan BCM, Robinson L, et al. A two decade dementia incidence comparison from the cognitive function and ageing studies I and II. Nat Commun 2016;7:11398.

40 Lobo A, Saz P, Marcos G, et al. Prevalence of dementia in a southern European population in two different time periods: the ZARADEMP project. Acta Psychiatr Scand 2007;116:299-307.

41 Central Statistics Office. Population and labour force projections, 2013. http://cso.ie/en/media/csoie/releasespublications/documents/ population/2013/poplabfor2016_2046.pdf.

42 Helmer C, Joly P, Letenneur L, et al. Mortality with dementia: results from a French prospective community-based cohort. Am J Epidemiol 2001:154:642-8.

43 Xie J, Brayne C, Matthews FE, et al. Survival times in people with dementia: analysis from population based cohort study with 14 year follow-up. BMJ 2008;336:258-62. 
44 Ganguli M, Dodge HH, Shen C, et al. Alzheimer disease and mortality: a 15-year epidemiological study. Arch Neurol 2005;62:779-84.

45 Koller D, Kaduszkiewicz $\mathrm{H}$, van den Bussche $\mathrm{H}$, et al. Survival in patients with incident dementia compared with a control group: a five-year follow-up. Int Psychogeriatr 2012;24:1522-30.

46 James BD, Leurgans SE, Hebert LE, et al. Contribution of Alzheimer disease to mortality in the United States. Neurology 2014;82:1045-50.

47 Tschanz JT, Corcoran C, Skoog I, et al. Dementia: the leading predictor of death in a defined elderly population: the Cache County study. Neurology 2004;62:1156-62.

48 Lewis FI, Torgerson PR. The current and future burden of late-onset dementia in the United Kingdom: estimates and interventions. Alzheimers Dement 2017:13:38-44.

49 Kopec JA, Finès P, Manuel DG, et al. Validation of population-based disease simulation models: a review of concepts and methods. BMC Public Health 2010;10:710.
50 Timmons S, Manning E, Barrett A, et al. Dementia in older people admitted to hospital: a regional multi-hospital observational study of prevalence, associations and case recognition. Age Ageing 2015;44:993-9.

51 Doblhammer G, Fink A, Zylla S, et al. Compression or expansion of dementia in Germany? an observational study of short-term trends in incidence and death rates of dementia between 2006/07 and 2009/10 based on German health insurance data. Alzheimers Res Ther 2015;7:66.

52 WHO. Draft global action plan on the public health response to dementia. Geneva: World Health Organsiation, 2016.

53 Pierse T, Keogh F, O'Shea E, et al. Geographic availability and accessibility of day care services for people with dementia in Ireland. BMC Health Serv Res 2020;20:476.

54 O'Shea E, Keogh F, Heneghan C. Post-Diagnostic support for people with dementia and their carers. Galway NUI Galway; 2018. 